\title{
3.5 POLARIZATION MEASUREMENTS OF OTHER PULSARS AT JODRELL BANK
}

\author{
D. A. GR AHA M \\ University of Manchester, Nuffield Radio Astronomy Laboratories, Jodrell Bank, U.K.
}

The polarization properties of a number of pulsars have been studied at Jodrell Bank using the $76 \mathrm{~m}$ Mark I radio telescope.

The feed system has outputs responding to the two senses of circular polarization, which are combined in the receiver to give the four Stokes parameters $I, V, Q$ and $U$. These are either displayed directly on an oscilloscope triggered in synchronism with the pulsar period, or integrated by an on-line computer. Some of these results are presented for comparison with the Crab Nebula pulsar.

Complex structure is frequently observed in single pulses, particularly in circular polarization. CP 0328 , however, shows a rather regular reversal of circular polarization in single pulses (Clark and Smith, 1969), still seen to a lesser degree in the average picture. The Stokes parameters $I$ and $V$ are shown in Figure 1 for two pulses recorded at $408 \mathrm{MHz}$, and also shows $I$ and $V$ for the same pulses at $240 \mathrm{MHz}$, having allowed for the dispersion delay of several periods. The similarity seen here between results at two different frequencies is mirrored by the integrated pulse results. Figure 2 shows integrated linear polarization for PSR 2045 at 408 and $240 \mathrm{MHz}$. Position angles at the lower frequency are displaced by a constant angle to emphasize the similarity. The swing of position angle in this source may be closely fitted by the geometrical model described by Radhakrishnan and Cooke (1969).

The variation of position angle through the pulse for six pulsars may be seen to better advantage in Figure 3, in which the intensity of linear polarization is shown by the length of a line, whose inclination from a constant but arbitrary zero is the position angle of linear polarization.

PSR 1929 is $100 \%( \pm 5 \%)$ polarized in the early part of the pulse, and about $70 \%$ in the latter half. Its position angle is seen to swing at a rate of about $1.3^{\circ}$ per degree of assumed pulsar rotation.

The net swing of position angle over the whole pulse for AP 1237 is seen to be almost zero, although some swing occurs in each of the two apparently separate double components.

The linear polarization picture for $200 \mathrm{~ms}$ of the period of CP 0950 at $408 \mathrm{MHz}$ clearly shows the interpulse, since, unlike the interpulse of NP 0532 , it is $100 \%$ $( \pm 20 \%$ ) polarized, and the average polarization of the main pulse is some $40 \%$.

Both early and late 'interpulses' are seen for CP 0328 at $408 \mathrm{MHz}$.

\section{References}

Clark, R. R. and Smith, F. G.: 1969, Nature 221, 747.

Radhakrishnan, V. and Cooke, D. J.: 1969, Astrophys. Letters 3, 223. 

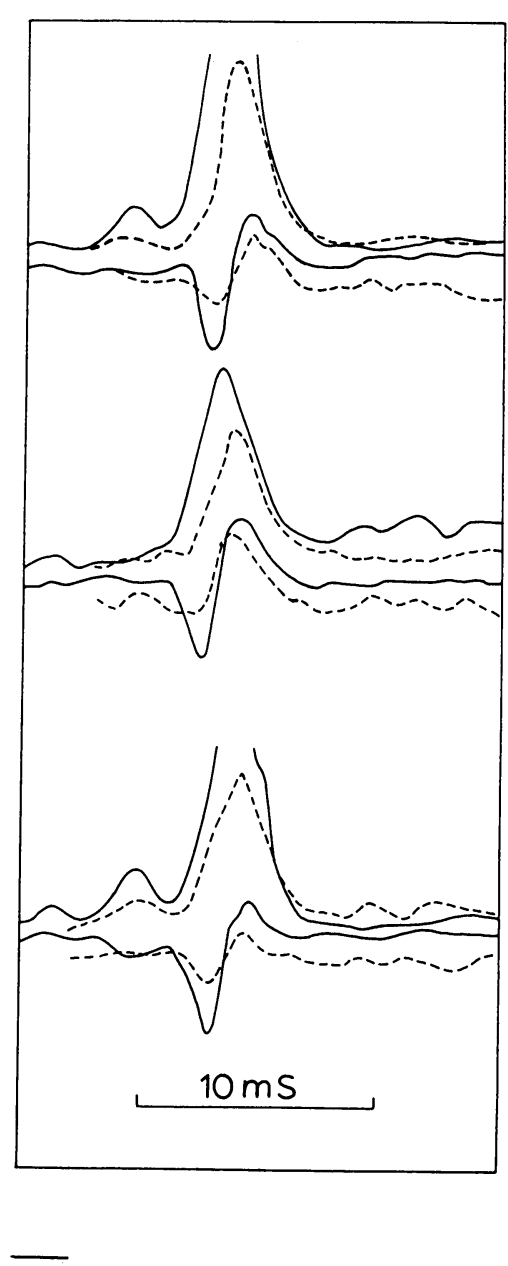

Fig. 1. Three pulses of CP 0328 in May 1970 recorded at $408 \mathrm{MHz}(-)$, $\Delta f=0.33 \mathrm{MHz}$ and $240 \mathrm{MHz}(-.-.-)$, $\Delta f=0.1 \mathrm{MHz}$. In each pulse the upper traces are Stokes parameter I (total intensity) and the lower traces are Stokes parameter V (circular).
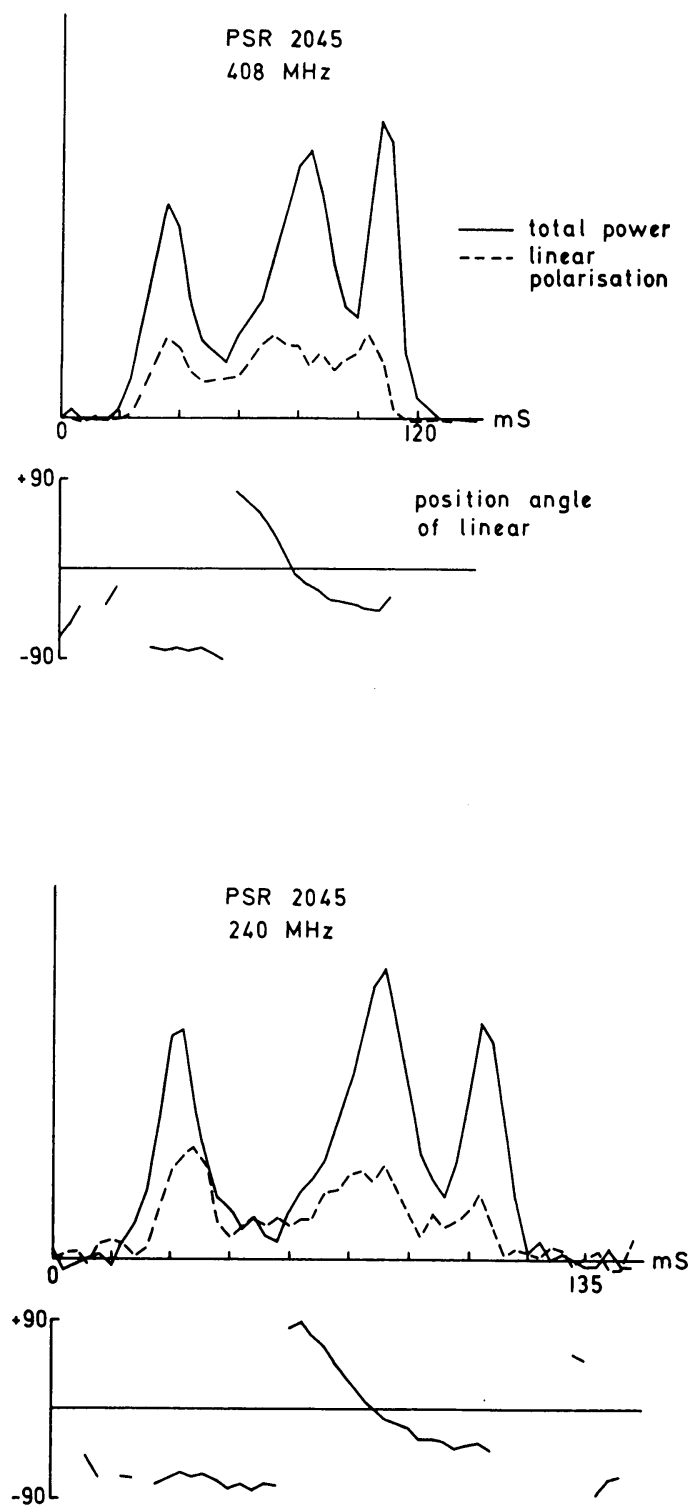

Fig. 2. The linear polarization of PSR 2045 at $408 \mathrm{MHz}$ and $240 \mathrm{MHz}$ integrated over many pulses. 


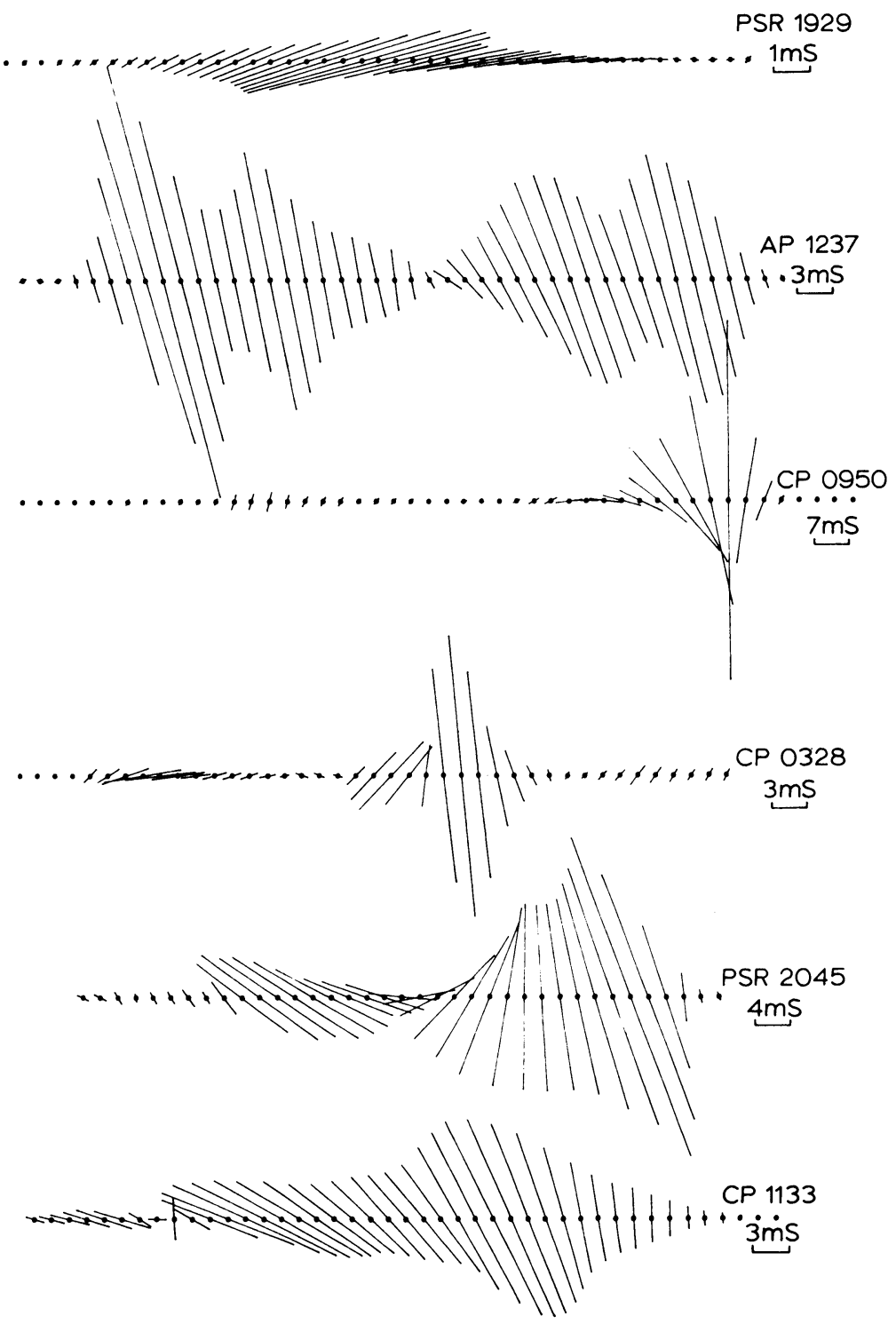

Fig. 3. The variation of the position angle of the linear polarization through the average pulse of six pulsars. 